\title{
Relations between pressure in pulmonary artery, left atrium, and left ventricle with special reference to events at end diastole
}

\author{
S. A. Forsberg ${ }^{1}$ \\ From the Medical Department I, Sahlgren's Hospital, University of Göteborg, Sweden
}

Results were extracted from 158 patients who have undergone diagnostic heart catheterization at rest. Seventeen were considered normal. Simultaneous pressure records from the pulmonary artery and left atrium were always made and often also from the left atrium and ventricle. Some of the main conclusions are as follows.

I) There is normally at rest left atrioventricular diastolic pressure congruence.

2) The $z$ point, that is the foot of the left atrial $c$ wave, is normally identical with the end-diastolic ventricular pressure.

3) Normally the pulmonary arterial diastolic pressure is approximately identical with the enddiastolic pressure of the left ventricle.

4) At the end of diastole, the flow and pressure gradient across the pulmonary vascular bed seem to be in phase and both are close to zero.

5) Patients with different cardiovascular diseases, the majority with mitral valvular disease, were compared with the normal group. With moderate mitral stenosis without much raised pulmonary vascular resistance, the relation between pulmonary arterial diastolic pressure and enddiastolic pressure in the left atrium is similar to that in normal patients.

Pulmonary arterial diastolic pressure has received relatively less attention than other measured entities in the pulmonary circulation such as the arterial systolic or mean pressure or pulmonary capillary venous pressure. The main purpose of the present study was to investigate factors determining the pulmonary diastolic pressure and its relation to events in the left ventricle and left atrium.

\section{Methods and material}

Results were extracted from the material of 158 patients who have undergone diagnostic right and left heart catheterization with measurement of the pulmonary blood volume. Details of the methods were described by Forsberg (1964).

Catheters were placed in the pulmonary artery and left atrium, and pressures were simultaneously recorded. In about one-third of the material the two pressures were recorded with the same zero and calibration pressure on congruent levels on the recording paper, giving a direct visual picture of the instantaneous pressure difference during the heart cycles. A similar technique was routinely used when left atrial and ventricular pressures were simultaneously recorded.

Received 23 September 1970.

1 Present address: Medicinska kliniken, Borås lasarett, 50115 Borås, Sweden.
The undamped natural frequency of the whole measuring system is of the order of $15-40$ Herz, the pulmonary arterial catheter usually giving values in the lower half of the range and the left atrial or left ventricular catheter rather more in the upper half. The degree of damping is around $0 \cdot 1$. An empirical comparison of the two recording systems is seen in Fig. I. In all figures the time between two thick lines equals $O \cdot I$ sec.

Calculations of average pressures were made out of to heart cycles. The end-diastolic pressure in the left atrium was measured from the $z$ point which is the foot of the $c$ wave. In absence of the c wave it was judged on the left atrium curve, from the left ventricular curve, or from the $R$ wave in the electrocardiogram. All results refer to investigations of patients at rest in the supine position.

The diagnoses in the total material were as follows: normal haemodynamics without heart or lung vascular disease (I5); obstructive lung disease with normal haemodynamics (2); left atrial myxoma (2); mitral valvular disease (8I); mitral and aortic valvular disease (13); aortic valvular disease (23); aortic coarctation (6); systemic hypertensive disease (I); cardiomyopathy (5); pulmonary stenosis (2); primary pulmonary hypertension (7); hyperkinetic heart syndrome (I). Absence of heart disease mostly implies patients with a systolic murmur where no pathology could be found. 

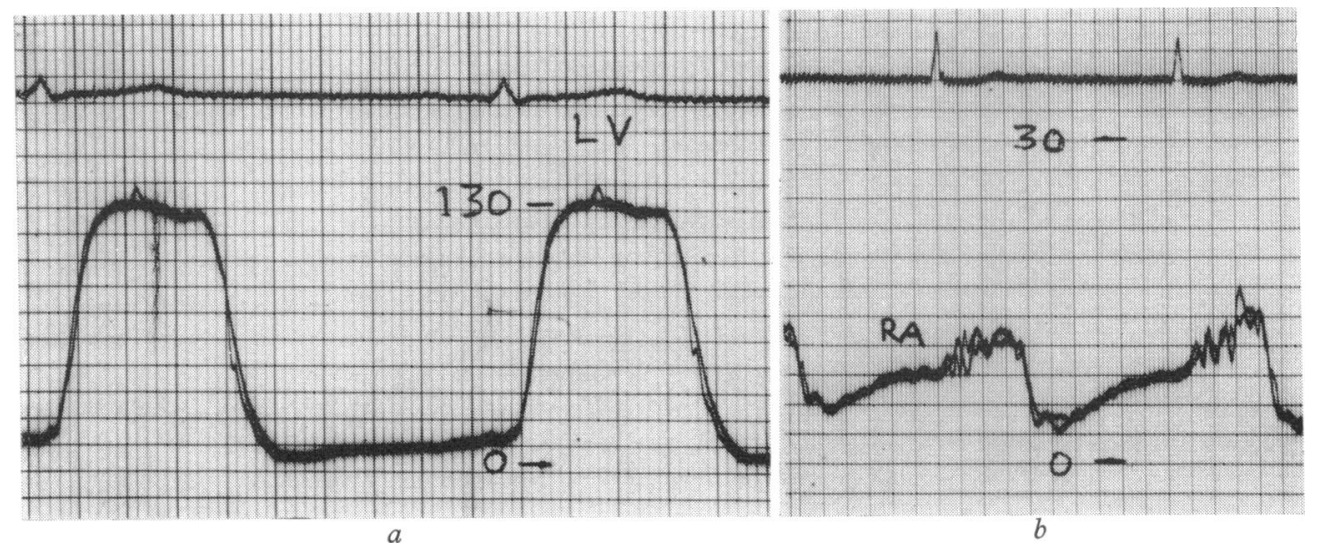

FIG. I (a) Two catheters in the left ventricle: diagnosis, a physiological murmur. (b) Two catheters in the right atrium: diagnosis, mitral stenosis.

\section{Results}

Relations between left atrial and left ventricular pressure during diastole and around end-diastolic point The atrioventricular gradient could be visually inspected in 8 patients without mitral obstruction: 7 had sinus rhythm ( 2 with normal hearts and 5 with some heart disease) and one had atrial fibrillation. With due regard to minor artefacts there was diastolic congruence in all patients (Fig. 2).

As the shape of the left atrial pressure curves generally agrees with that previously reported, only some points of interest should be stressed. The upstroke of the $c$ wave is consistently congruent with the upstroke of ventricular systole in patients with sinus rhythm and normal haemodynamics or some ventricular or aortic disease (Fig. 2). This fact serves as a test of validity of our recordings and verifies the atrial $\mathrm{z}$ point as an indicator of the end-diastolic ventricular pressure.

The $c$ wave is, however, not consistent. In I7 haemodynamically normal patients without heart disease, 6 lacked a c wave and 2 had a c wave in one recording but not in another (Fig. 2b).

In patients with mitral valvular disease and atrial fibrillation a c wave is always present, beginning at the point of equilibration of left atrial and ventricular pressure during the isovolumetric ventricular contraction phase (Fig. 3).

Relations between pulmonary arterial diastolic and left atrial pressure The 17 patients with normal haemodynamics without signs of heart disease were studied separately. The mean left atrial pressure varied from 3 to
I2 $\mathrm{mmHg}$ with a mean of $6.4 \mathrm{~mm}$. The difference between pulmonary arterial diastolic and mean left atrial pressure is $1 \cdot 4 \pm 2 \cdot 0$ mmHg. Pulmonary arterial diastolic pressure minus left atrial end-diastolic pressure is $-0.4 \pm \mathrm{I} .5 \mathrm{mmHg}$. The pulmonary arterial pressure decreases during diastole and reaches its minimum at a level equal to the peak of the left atrial a wave or slightly below this peak, constituting a pressure gradient from the atrium to the pulmonary artery for a moment (Fig. 4).

A selection was made of patients having heart disease, atrial fibrillation, and a mean left atrial pressure at rest of $12 \mathrm{~mm}$ or less and a pulmonary vascular resistance less than 2 units. It happened that all is such patients had mitral valvular disease. Pulmonary arterial diastolic pressure minus left atrial mean pressure is $\mathrm{I} \cdot 0 \pm 2.0 \mathrm{mmHg}$. Pulmonary arterial diastolic pressure minus left atrial end-diastolic pressure is $2.5 \pm 1.5 \mathrm{mmHg}$. These results are close to those in the normal patients (Fig. 5).

During long systolic intervals, the pulmonary arterial pressure can become congruent with the left atrial pressure (Fig. 5b), but the left atrial pressure was never highest at the end of diastole with atrial fibrillation.

The Table shows the difference between pulmonary arterial diastolic pressure and left atrial mean pressure related to the pulmonary vascular resistance in all patients with sinus rhythm or atrial fibrillation. These two groups behaved similarly and were therefore combined.

For each increment of pulmonary vascular resistance the average pressure difference increased but not more than $4 \mathrm{mmHg}$ with 

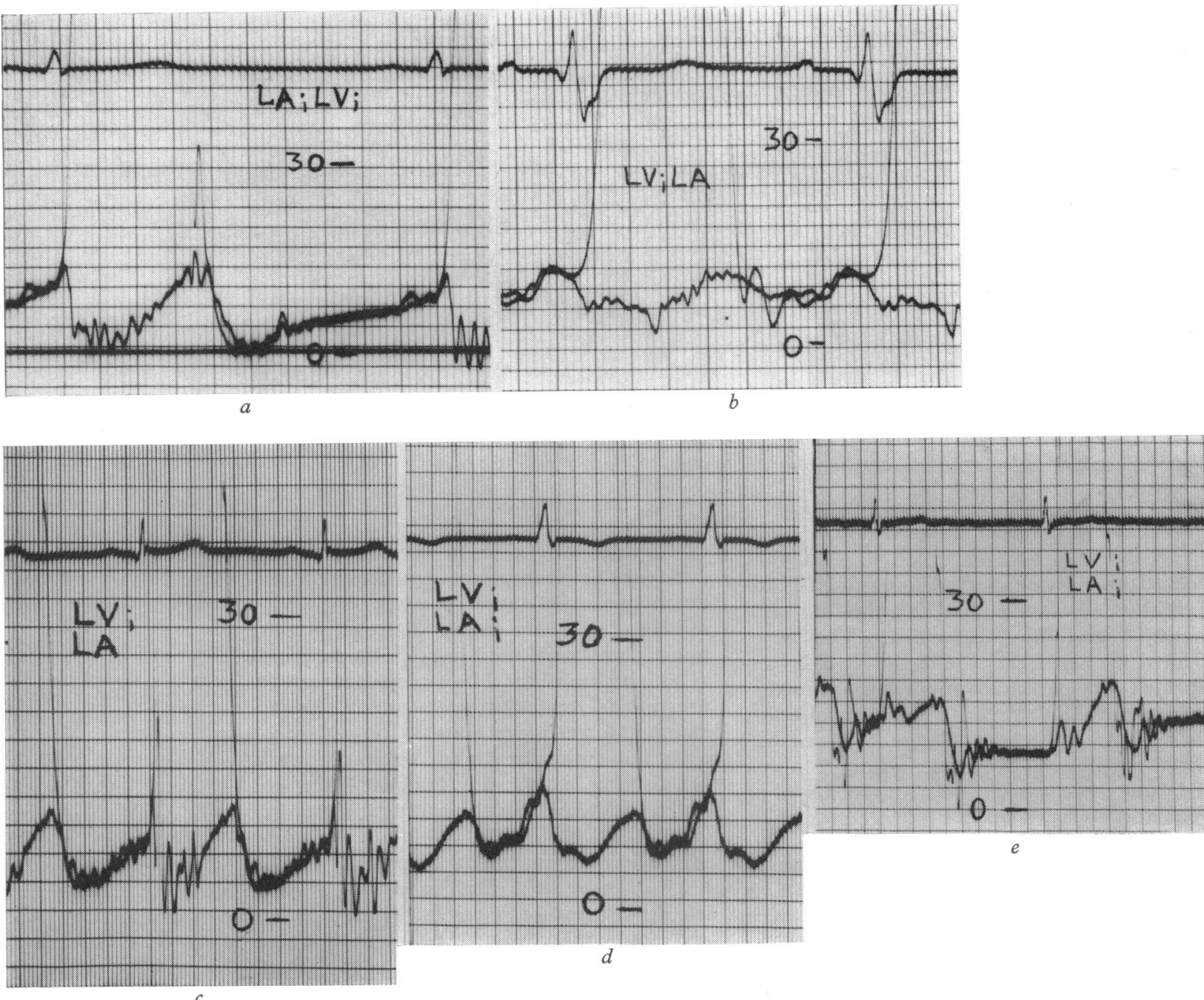

FIG. 2 Simultaneous records from the left atrium and left ventricle in 2 normal patients $(a$ and $b)$ and patients with cardiovascular disease but normal mitral orifice $(c, d$, and $e$ ).

TABLE 155 patients with sinus rhythm or atrial fibrillation ( 17 without and 138 with some cardiovascular disease)

\begin{tabular}{lll}
\hline $\begin{array}{l}\text { Pulm. vasc. } \\
\text { resist. (units) }\end{array}$ & $\begin{array}{l}\text { No. of } \\
\text { patients }\end{array}$ & $\begin{array}{l}\text { Pulm. art. diast. - } \\
\text { left atrial mean } \\
(m m H g)\end{array}$ \\
\hline $0 \cdot 0-0 \cdot 9$ & 27 & $\pm 0(-4 ;+4)$ \\
$1 \cdot 0-1 \cdot 9$ & 83 & $+2(-3 ;+9)$ \\
$2 \cdot 0-2 \cdot 9$ & 22 & $+4(-1 ;+16)$ \\
$3 \cdot 0-3 \cdot 9$ & 8 & $+4(-1 ;+8)$ \\
$4 \cdot 0-4 \cdot 9$ & 4 & $+5(+1 ;+8)$ \\
$5 \cdot 0-5 \cdot 9$ & 1 & $+7((-)$ \\
$6 \cdot 0-6 \cdot 9$ & 2 & $+27(+26 ;+27)$ \\
$7 \cdot 0-7 \cdot 9$ & 2 & $+9(0 ;+18)$ \\
$>8 \cdot 0$ & 6 & $+36(+19 ;+63)$ \\
\hline
\end{tabular}

* Pulmonary vascular resistance expressed in units (pressure difference $\mathrm{mmHg}$, cardiac output in $1 . / \mathrm{min}$ ). Within brackets are the extreme values in each group, and outside are the mean values. resistance as high as 4 units. Individual variations exist among those with heart disease, some with a low resistance and large pressure gradient, others with a high resistance and a small gradient.

In the 24 patients with pulmonary vascular resistance between $\mathrm{I} \cdot 0$ and $\mathrm{I} \cdot 9$ units, those 12 with the largest pressure gradient (pulmonary diastolic minus left atrial mean pressure) were compared with those 12 with the lowest gradient. On an average the largest difference was associated with a higher heart rate and lower mean left atrial pressure, whereas the stroke volume and pulmonary diastolic pressure were similar in the two groups. 

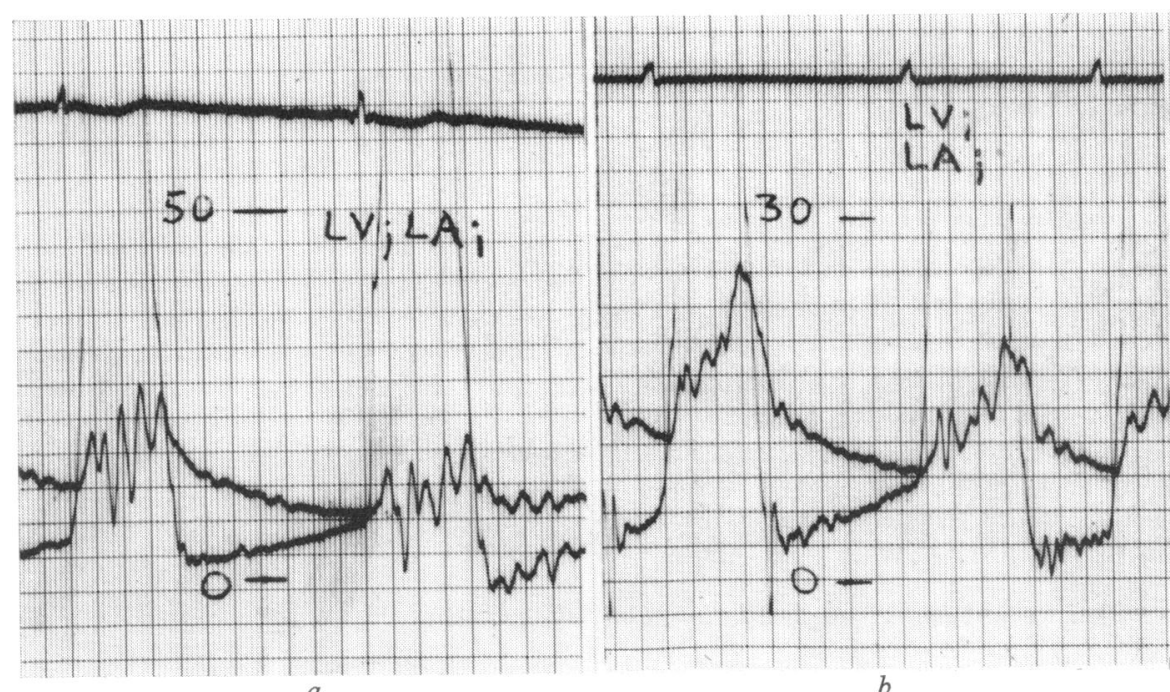

$b$

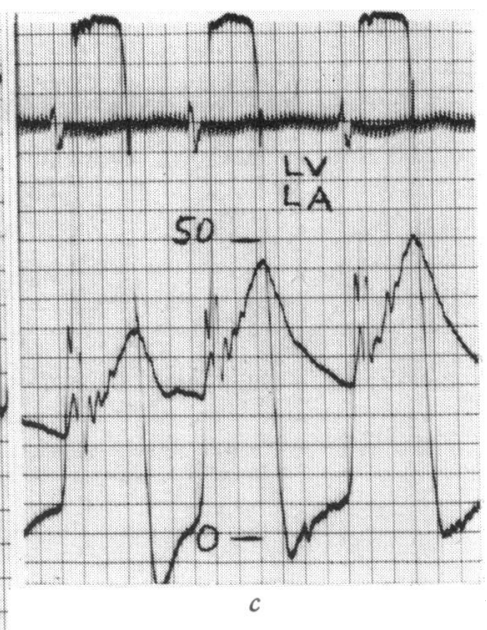

FIG. 3 Simultaneous records from the left atrium and left ventricle in patients with mitral stenosis and atrial fibrillation.

\section{Discussion}

A review of normal pressure curves from the pulmonary artery, left atrium, and left ventricle, and their interrelations and connexions to haemodynamic events was presented by Brecher and Galletti (1963). Left atrioventricular pressure events in mitral valvular disease have been repeatedly reported (Braunwald et al., 1955; Gordon, Kirschner and Moscovits, I961; Wooley et al., 1968). Our recordings in patients with normal as well as abnormal haemodynamics agree with those previously described. Pertinent to this study is the conclusion that in the resting condition there is normally congruence during the whole of diastole between the left atrial and ventricular pressure. In mitral valvular disease with moderate or slight stenosis it also comes to a congruence if the gradient disappears before the end of diastole.

Our results show that the end-diastolic left ventricular pressure normally is the factor which sets the pulmonary arterial diastolic pressure at rest. In about 95 per cent the pressure difference should be less than 3 mmHg. Recently, Bouchard, Gault, and Ross (I969) presented similar data at a meeting. In our patients with mitral valvular disease and normal to slightly increased pulmonary vascular resistance the figures were close to

FIG. 4 Records from patients with normal hearts. Only the tracings from the pulmonary artery and left atrium should be considered.

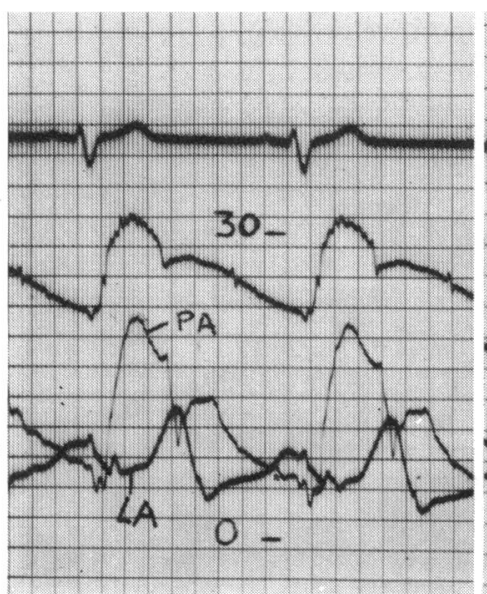

$a$

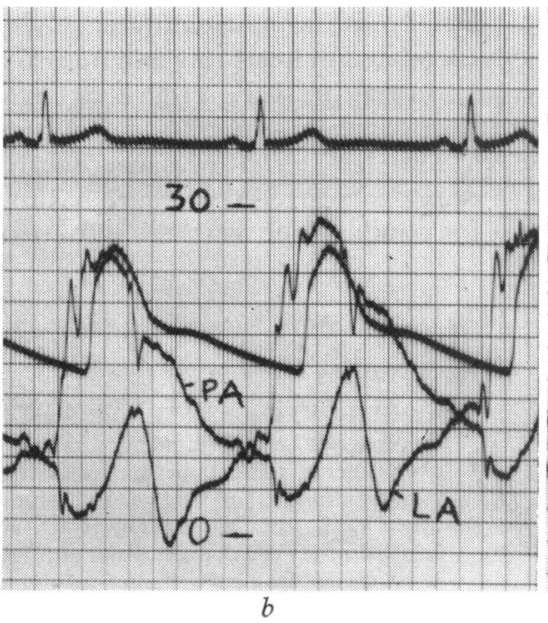

b

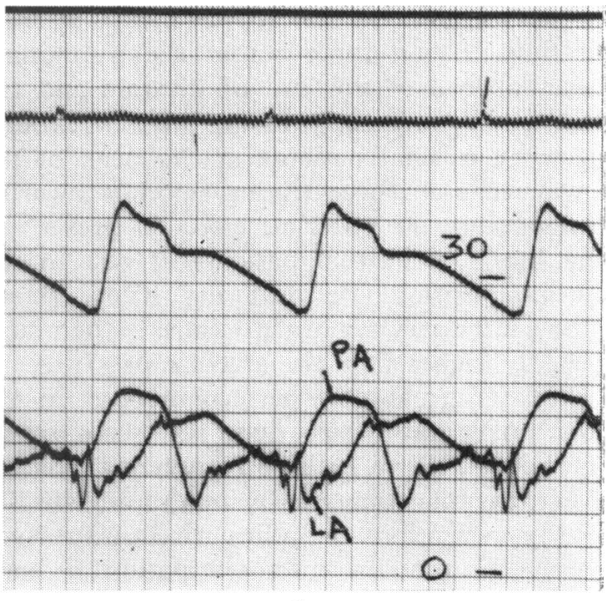




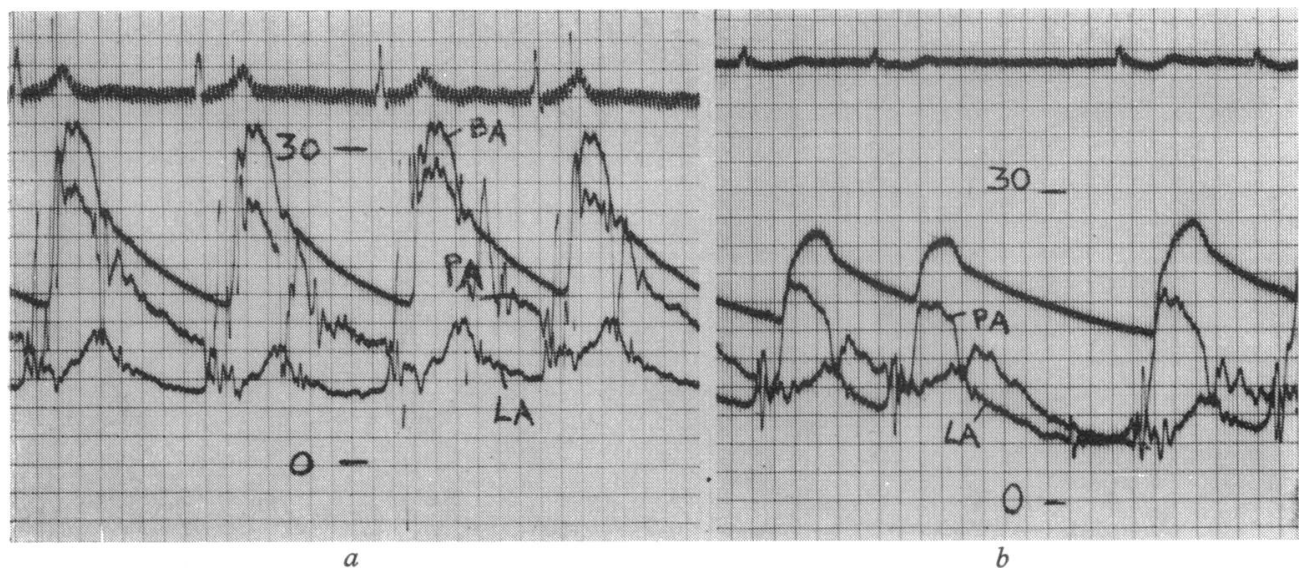

FI G. 5 Records from patients with mitral stenosis and atrial fibrillation. Only the tracings from the pulmonary artery and left atrium should be considered.

those in normal patients. From our data on the normal patients, it can be calculated that the difference between mean and end-diastolic left atrial pressure is $\mathrm{I} \cdot 8 \mathrm{~mm}$. Braunwald et al. (I96I) found $0.3 \mathrm{~mm}$ and Samet et al. (I965) $\mathrm{I} \cdot 0 \mathrm{~mm}$.

These facts have important implications for patients where the pulmonary arterial pressure is continually monitored in order to disclose left ventricular insufficiency and the wedged pressure cannot be easily recorded. This is now becoming more common in coronary care units. It is necessary to do future studies similar to the present one and to include patients with a relatively normal vascular bed afflicted by acute ventricular failure.

Our unpublished observations have failed to show any significant phase shift of the pressure curve, withdrawing the catheter from the peripheral lung vessels to the pulmonary arterial stem or to the left atrium by the transseptal route. Identity of pressure in the pulmonary artery and left atrium means absence of flow-driving force across the pulmonary vascular bed at the end of diastole and the momentary reversed pressure difference found in some normal patients at the peak of the a wave means a force driving blood backwards. Presumably no waterfall effect is present in the lungs of normal patients at rest in the supine position (Permutt and Riley, 1963). The pulmonary venous pressure is then supposedly higher than the alveolar pressure.

What normal flow pattern there is in the pulmonary capillaries does not seem to be settled yet. From Fishman's review (1963), it is evident that some plethysmographic studies and investigations including direct inspection of the pulmonary microcirculation in vivo support an intermittent type of flow whereas other plethysmographic studies support a continuous but pulsatile flow.

Cineradiographic observations of radiopaque droplets in the pulmonary veins and left atrium of dogs have demonstrated flow with a small momentary backward component occurring close to the end-diastolic point (Ferrario, Nordenström, and Paulin, I968). Direct flow measurements from the pulmonary vein and left atrium are in accordance with these results (Morkin et al., 1965).

The sum of evidence is consistent with the idea that the flow through the pulmonary veins and the pressure gradient across the whole pulmonary vascular bed are in approximate phase at the end of diastole. Both are minimal and at least sometimes momentarily reversed.

Our patients with pulmonary vascular resistance between I and 2 units had normal as well as abnormal pulmonary vascular beds. The end-diastolic pulmonary arterial to left atrial mean pressure difference was positively correlated to heart rate and negatively to mean left atrial pressure. High heart rate implies a short time for equilibration of pressures, and a low left atrial pressure means that the pulmonary arterial pressure has to fall deeper for equilibration with the left atrium.

\section{References}

Bouchard, R. J., Gault, J. H., and Ross, J., Jr. (1969). Comparison of pulmonary arterial end-diastolic pressures in patients with and without left ventricular disease. Circulation, 40, Suppl. 3, p. 49.

Braunwald, E., Brockenbrough, E. C., Frahm, C. J., and Ross, J. (196r). Left atrial and left ventricular pressures in subjects without cardiovascular disease. Circulation, 24, 267. 
Braunwald, E., Moscovitz, H. L., Amram, S. S., Lasser, R. P., Sapin, S. O., Himmelstein, A., Ravitch, M. M., and Gordon, A. J. (I955). The hemodynamics of the left side of the heart as studied by simultaneous left atrial, left ventricular, and aortic pressures; particular reference to mitral stenosis. Circulation, 12, 69.

Brecher, G. A., and Galletti, P. M. (1963). Functional anatomy of cardiac pumping. In Handbook of Physiology, Section 2: Circulation, Vol. 2, pp. 759798. American Physiology Society, Washington, D.C.

Ferrario, C. M., Nordenström, B., and Paulin, S. (1968). Flow velocity variations in the pulmonary veins of the dog. Investigative Radiology, 3, 73.

Fishman, A. P. (1963). Dynamics of the pulmonary circulation. In Handbook of Physiology, Section 2: Circulation, Vol. 2, pp. 1667-1743. American Physiology Society, Washington, D.C.
Forsberg, S. A. (1964). Pulmonary blood volume in man. Acta Medica Scandinavica, 175, Suppl. 410. Gordon, A. J., Kirschner, P. A., and Moscovits, H. L. (1961). Hemodynamics of Aortic and Mitral Valve Disease. Grune and Stratton, New York.

Morkin, E., Collins, J. A., Goldman, H. S., and Fishman, A. P. (1965). Pattern of blood flow in the pulmonary veins of the dog. Fournal of Applied Physiology, 20, II 18.

Permutt, S., and Riley, R. L. (1963). Hemodynamics of collapsible vessels with tone: the vascular waterfall. Fournal of Applied Physiology, 18, 924.

Samet, P., Bernstein, W. H., Medow, A., and Levine, S. (1965). Transseptal left heart dynamics in thirtytwo normal subjects. Diseases of the Chest, 47, 632 .

Wooley, C. F., Klassen, K. P., Leighton, R. F., Goodwin, R. S., and Ryan, J. M. (1968). Left atrial and left ventricular sound and pressure in mitral stenosis. Circulation, 38,'295. 